\title{
Desastres, crecimiento económico y respuesta fiscal en los países de América Latina y el Caribe, 1972-2010
}

\author{
Omar D. Bello
}

\section{Resumen}

El objetivo de este trabajo es estimar el impacto de los desastres climáticos y geológicos en la tasa de crecimiento del producto interno bruto (PIB) per cápita y la tasa de crecimiento del gasto fiscal per cápita de los países de América Latina y el Caribe. Los resultados indican que hay efectos diferenciados por tipo de desastre y por subregiones. En los países del Caribe, la respuesta típica de la tasa de crecimiento del PIB per cápita a los desastres climáticos fue negativa. Por su parte, la respuesta a un desastre de origen geológico no fue estadísticamente significativa. En los países de Centroamérica, la respuesta de la tasa de crecimiento del PIB per cápita a los desastres climáticos fue negativa el primer año y positiva el tercer año, mientras que la respuesta a los desastres de origen geológico fue positiva en el segundo y tercer año.

\section{Palabras clave}

Desastres naturales, crecimiento económico, producto interno bruto, gastos públicos, América Latina y el Caribe

Clasificación JEL

Q54, L6, L7, L8

Autor

Omar D. Bello es Coordinador de la Unidad de Desarrollo Sostenible y Evaluación de Desastres de la sede subregional de la Comisión Económica para América Latina y el Caribe (CEPAL) para el Caribe, Puerto España. omar.bello@cepal.org

El autor agradece los comentarios y las sugerencias de Jean Acquatella, Adriana Arreaza, Carlos de Miguel, Leda Peralta y Omar Zambrano, así como la excelente asistencia de Ignacio Cristi LeFort y Viviana Rosales Salinas para esta investigación. Como es usual en estos casos, todas las opiniones, errores u omisiones son de exclusiva responsabilidad del autor. 


\section{Introducción}

Los desastres son perturbaciones que podrían afectar a distintas variables ambientales, económicas y sociales de un país ${ }^{2}$. Son fenómenos de ocurrencia común y cuya frecuencia se ha incrementado a lo largo del tiempo ${ }^{3}$. Según la Base de Datos Internacional sobre Desastres (EM-DAT) de la Oficina de Asistencia de Desastres en el Extranjero y el Centro de Investigación sobre la Epidemiología de los Desastres (OFDA/CRED) de la Universidad Católica de Lovaina en Bruselas, que cuenta con el registro más extenso de desastres a nivel mundial, en el período 1970-2010 tuvieron lugar 10.271 desastres en el mundo, de los cuales un $23,7 \%$ se produjeron en América (16,9\% en países de América Latina y el Caribe), mientras que Asia fue el continente más afectado (39,3\% de los desastres ocurrieron en esa región). Dicha base de datos define a un desastre como un evento que cumple uno de los siguientes criterios: i) se informa de diez o más muertes; ii) se informa que 100 o más personas resultaron afectadas; iii) se declara un estado de emergencia, o iv) se hace una petición de ayuda.

Según esa fuente, en el mencionado período ocurrieron 1.737 desastres en América Latina y el Caribe, de los cuales 1.392 fueron de origen climático, 227 de origen geológico y 118 de origen biológico. En lo que se refiere a las subregiones, el crecimiento más importante del número de desastres ocurrió en Centroamérica (410\%) y en el Caribe (372\%) Este comportamiento estuvo determinado por la ocurrencia de fenómenos de origen climático, que se incrementaron un $448 \%$ y un $438 \%$ en Centroamérica y en el Caribe, respectivamente. La evidencia que se presenta en la segunda sección de este artículo indica que los desastres en estas subregiones han sido más intensos en términos de muertes, población afectada y daños materiales.

Los desastres se suman a las variadas perturbaciones que afectan a las economías de la región, entre ellas, los ciclos de negocios de los países de altos ingresos - cuyas repercusiones en el comercio internacional suponen cambios tanto en el volumen exportado, sobre todo de productos básicos, como en sus precios ${ }^{6}$ - y las perturbaciones en los mercados financieros internacionales. Así, en los últimos 40 años han tenido lugar distintas crisis como las de la deuda, la de ahorro y préstamo de los Estados Unidos, la mexicana, la asiática, la argentina y la del mercado inmobiliario de los Estados Unidos, que han afectado a los países de América Latina y el Caribe a través de diferentes mecanismos (véanse, por ejemplo, Titelman, Pérez y Pineda (2009), De Gregorio y Valdés (2001) y Reinhart y Rogoff (2008)). Está bien documentado que las caídas del producto interno bruto (PIB) de los países desarrollados, de los precios de los productos básicos de exportación y de la tasa de interés externa son factores que permiten explicar, entre otros, diversos episodios que han afectado el desempeño económico de los países de América Latina y el Caribe ${ }^{7}$.

Desde el punto de vista económico, los desastres tienen efectos e impactos. Los primeros se refieren a la afectación de acervos, es decir a los daños, y a las alteraciones de los flujos, que son las pérdidas y los costos adicionales. Los impactos son consecuencias de los efectos en distintas

2 En este artículo se usa el término "desastres", ya que estas situaciones son producto de un evento natural (por ejemplo, un terremoto, una erupción volcánica o un huracán) y de una vulnerabilidad generada por el ser humano. Por ello, desde hace años la comunidad que analiza el tema ha sustituido el término "desastres naturales" por "desastres".

3 Según Stromberg (2007), el incremento del número de desastres está ligado a tres factores: i) la información sobre desastres pequeños ha mejorado en los últimos años; ii) la población mundial se ha incrementado, por lo que una mayor cantidad de personas están expuestas a amenazas naturales, y iii) los eventos climáticos son más frecuentes.

4 Centroamérica incluye a Belice, Costa Rica, El Salvador, Guatemala, Honduras, Nicaragua y Panamá; el Caribe incluye a Antigua y Barbuda, las Bahamas, Barbados, Cuba, Dominica, Granada, Haití, Jamaica, República Dominicana, Saint Kitts y Nevis, Santa Lucía, San Vicente y las Granadinas y Trinidad y Tabago.

5 América del Sur incluye a la Argentina, Bolivia (Estado Plurinacional de), el Brasil, Chile, el Ecuador, Guyana, el Paraguay, el Perú, Suriname y Venezuela (República Bolivariana de).

6 Con excepción de México y el Brasil, en el período 2000-2010 más del 45\% de la canasta de exportación media anual de los países de América Latina correspondió a productos básicos.

7 Laeven y Valencia (2008) señalan que de las 41 crisis bancarias sobre las que informan, 13 tuvieron lugar en América Latina. 
variables sociales y económicas, como los ingresos de las familias, el desempleo, el crecimiento del PIB y el déficit fiscal, entre otras ${ }^{8}$. Así, cabe esperar que la destrucción de acervo por un desastre interrumpa temporalmente los flujos de producción y afecte las finanzas públicas debido, por ejemplo, a la menor recaudación impositiva y a los costos adicionales que podría generar la emergencia.

Este artículo se centra en la estimación de los impactos de diferentes tipos de desastres en dos variables: la tasa de crecimiento del PIB per cápita y la tasa de crecimiento del gasto fiscal per cápita en los países de América Latina y el Caribe, así como en las dos subregiones más intensamente afectadas por estos eventos, el Caribe y Centroamérica.

El presente artículo se organiza de la siguiente manera: a continuación, en la segunda sección, se presentan los hechos estilizados de los desastres ocurridos en América Latina y el Caribe; en la tercera sección figura una revisión de la literatura sobre el impacto de los desastres en la actividad económica, las finanzas públicas y las variables sociales; en la cuarta sección se presentan la metodología de estimación y las variables utilizadas; en la quinta sección se incluyen las funciones impulso-respuesta (FIR) estimadas de la tasa de crecimiento del PIB per cápita y de la tasa de crecimiento del gasto del gobierno per cápita ante perturbaciones en las variables examinadas. Por último, se presentan algunas evaluaciones de los resultados.

\section{Hechos estilizados de los desastres ocurridos en América Latina y el Caribe}

En esta sección se analizan la evolución de los desastres y algunas de las medidas de intensidad normalmente utilizadas, como las muertes, la población afectada y los daños. El énfasis está puesto en los desastres ocurridos en América Latina y el Caribe.

\section{Número de desastres}

El número de desastres se ha incrementado en todos los continentes durante el período analizado 9 . Este comportamiento se verificó para todos los tipos de desastres, siendo los de origen climático los que más aumentaron (véase el gráfico 1) ${ }^{10}$. En el caso de los desastres climáticos se estableció la siguiente clasificación: i) tormentas y ii) otros desastres climáticos, entre los que se incluyen inundaciones, sequías y movimientos de masa húmeda. En el cuadro 1 se presenta la dinámica de estos eventos por décadas en América Latina y el Caribe y se observa que tanto las tormentas como los otros desastres climáticos crecieron ininterrumpidamente ${ }^{11}$. Nótese que entre la década de 1970 y la de 2000 los desastres climáticos aumentaron un 326\% debido a la evolución de las tormentas, que se incrementaron un $453 \%$. En las cifras subregionales incidió lo acontecido en el Caribe y en Centroamérica, donde las tormentas crecieron un 540\% y un 533\%, respectivamente, y otros desastres climáticos aumentaron un 309\% y un 425\%, respectivamente.

\footnotetext{
8 Véase CEPAL (2014).

9 Véase Stromberg (2007).

${ }^{10}$ La clasificación utilizada corresponde a la de Skidmore y Toya (2002).

${ }^{11}$ Con el objeto de reducir el ruido que podría presentarse como resultado de la consideración de desastres particulares, se presenta la información por décadas, específicamente las cuatro décadas comprendidas entre 1970 y 1999.
} 


\section{Gráfico 1}

Desastres según su origen climático o geológicos, 1970-2010

(En número de desastres)

A. Mundo

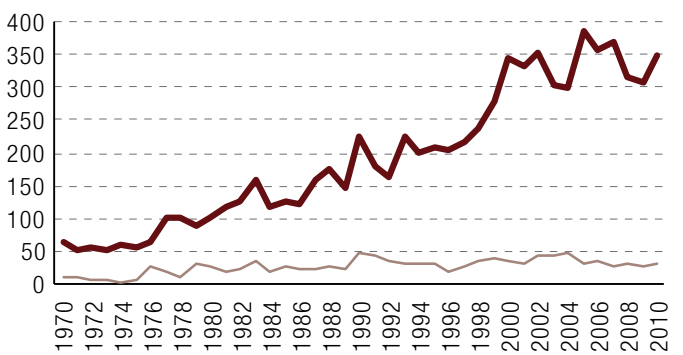

B. América Latina y el Caribe ${ }^{a}$

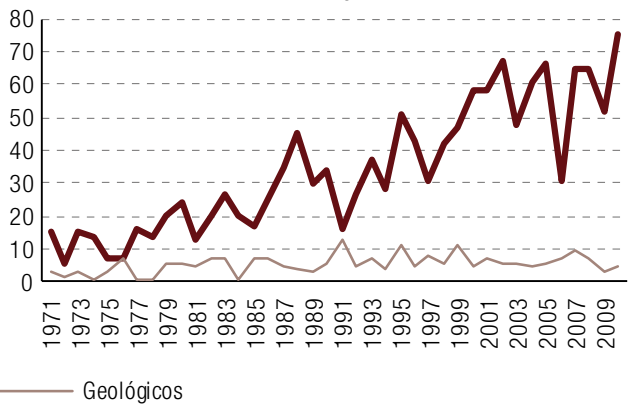

Fuente: Base de Datos Internacional sobre Desastres (EM-DAT) de la Oficina de Asistencia de Desastres en el Extranjero y el Centro de Investigación sobre la Epidemiología de los Desastres (OFDA/CRED) de la Universidad Católica de Lovaina.

a América Latina y el Caribe incluye: Antigua y Barbuda, Argentina, Bahamas, Barbados, Belice, Bolivia (Estado Plurinacional de), Brasil, Chile, Colombia, Costa Rica, Cuba, Dominica, Ecuador, El Salvador, Granada, Guatemala, Guyana, Haití, Honduras, Jamaica, México, Nicaragua, Panamá, Paraguay, Perú, República Dominicana, Saint Kitts y Nevis, Santa Lucía, San Vicente y las Granadinas, Suriname, Trinidad y Tabago, Uruguay y Venezuela (República Bolivariana de).

\section{Cuadro 1}

América Latina y el Caribe: desastres por tipo de evento y décadas

(En número de desastres)

\begin{tabular}{|c|c|c|c|c|}
\hline Décadas & Tormentas & $\begin{array}{l}\text { Otros desastres de } \\
\text { origen climático }\end{array}$ & $\begin{array}{l}\text { Desastres de origen } \\
\text { geológico }\end{array}$ & Total \\
\hline \multicolumn{5}{|c|}{ América Latina y el Caribe } \\
\hline 1970-1979 & 34 & 100 & 30 & 164 \\
\hline 1980-1989 & 70 & 186 & 52 & 308 \\
\hline 1990-1999 & 130 & 226 & 76 & 432 \\
\hline $2000-2009$ & 188 & 383 & 62 & 633 \\
\hline Total & 422 & 895 & 220 & 1537 \\
\hline \multicolumn{5}{|l|}{ El Caribe $^{b}$} \\
\hline 1970-1979 & 15 & 11 & 4 & 30 \\
\hline 1980-1989 & 42 & 38 & 0 & 80 \\
\hline 1990-1999 & 67 & 30 & 6 & 103 \\
\hline $2000-2009$ & 96 & 45 & 7 & 148 \\
\hline Total & 220 & 124 & 17 & 361 \\
\hline \multicolumn{5}{|l|}{ Centroamérica $^{\mathrm{C}}$} \\
\hline 1970-1979 & 6 & 16 & 11 & 33 \\
\hline 1980-1989 & 6 & 21 & 23 & 50 \\
\hline 1990-1999 & 21 & 39 & 37 & 97 \\
\hline 2000-2009 & 38 & 84 & 21 & 143 \\
\hline Total & 71 & 160 & 92 & 323 \\
\hline
\end{tabular}

Fuente: Base de Datos Internacional sobre Desastres (EM-DAT) de la Oficina de Asistencia de Desastres en el Extranjero y el Centro de Investigación sobre la Epidemiología de los Desastres (OFDA/CRED) de la Universidad Católica de Lovaina.

a América Latina y el Caribe incluye: Antigua y Barbuda, Argentina, Bahamas, Barbados, Belice, Bolivia (Estado Plurinacional de), Brasil, Chile, Colombia, Costa Rica, Cuba, Dominica, Ecuador, El Salvador, Granada, Guatemala, Guyana, Haití, Honduras, Jamaica, México, Nicaragua, Panamá, Paraguay, Perú, República Dominicana, Saint Kitts y Nevis, Santa Lucía, San Vicente y las Granadinas, Suriname, Trinidad y Tabago, Uruguay y Venezuela (República Bolivariana de).

b El Caribe incluye: Antigua y Barbuda, Bahamas, Barbados, Cuba, Dominica, Granada, Haití, Jamaica, República Dominicana, Saint Kitts y Nevis, Santa Lucía, San Vicente y las Granadinas y Trinidad y Tabago.

c Centroamérica incluye: Belice, Costa Rica, El Salvador, Guatemala, Honduras, Nicaragua y Panamá. 


\section{Muertes}

De todas las medidas de intensidad de desastres, esta es la que se ve más influenciada por eventos particulares. En el período 1970-2010 fallecieron en el mundo 3.450.255 personas a consecuencia de desastres ${ }^{12}$. Del total, 498.030 muertes ocurrieron en América Latina y el Caribe. Nótese que un $73,1 \%$ de esos fallecimientos ocurrieron como resultado de cinco eventos ${ }^{13}$ : i) el terremoto en Chimbote (Perú, 1970); ii) el terremoto en Ciudad de Guatemala (1976); iii) la erupción del volcán Nevado del Ruiz (Colombia, 1985); iv) el deslave en Vargas (República Bolivariana de Venezuela, 1999), y v) el terremoto de Puerto Príncipe (2010) ${ }^{14}$. En el cuadro 2 se muestra la evolución del número de muertes por cada 1.000 habitantes por décadas. Nótese que a lo largo de estas décadas, en América Latina y el Caribe se alternan aumentos y disminuciones del número de fallecimientos. Cabe destacar que, en lo que se refiere a esta medida de intensidad, el Caribe y Centroamérica han superado a toda la región en cada década.

Cuadro 2

América Latina y el Caribe: muertes por desastres, por décadas

(En número de muertes por cada 1.000 habitantes)

\begin{tabular}{lccc}
\hline Década & América Latina y el Caribe $^{\mathrm{a}}$ & El Caribe $^{\mathrm{b}}$ & Centroamérica $^{\mathrm{c}}$ \\
\hline $1970-1979$ & 0,063 & 0,011 & 0,452 \\
\hline $1980-1989$ & 0,014 & 0,007 & 0,015 \\
\hline $1990-1999$ & 0,017 & 0,008 & 0,065 \\
\hline $2000-2009$ & 0,004 & 0,021 & 0,012 \\
\hline $2010-2011$ & 0,403 & 8,914 & 0,014 \\
\hline
\end{tabular}

Fuente: Base de Datos Internacional sobre Desastres (EM-DAT) de la Oficina de Asistencia de Desastres en el Extranjero y el Centro de Investigación sobre la Epidemiología de los Desastres (OFDA/CRED) de la Universidad Católica de Lovaina.

a América Latina y el Caribe incluye: Antigua y Barbuda, Argentina, Bahamas, Barbados, Belice, Bolivia (Estado Plurinacional de), Brasil, Chile, Colombia, Costa Rica, Cuba, Dominica, Ecuador, El Salvador, Granada, Guatemala, Guyana, Haití, Honduras, Jamaica, México, Nicaragua, Panamá, Paraguay, Perú, República Dominicana, Saint Kitts y Nevis, Santa Lucía, San Vicente y las Granadinas, Suriname, Trinidad y Tabago, Uruguay y Venezuela (República Bolivariana de).

b El Caribe incluye: Antigua y Barbuda, las Bahamas, Barbados, Cuba, Dominica, Granada, Haití, Jamaica, República Dominicana, Saint Kitts y Nevis, Santa Lucía, San Vicente y las Granadinas y Trinidad y Tabago.

c Centroamérica incluye: Belice, Costa Rica, El Salvador, Guatemala, Honduras, Nicaragua y Panamá.

Durante los años setenta y ochenta, el $81 \%$ de las muertes correspondió a desastres de origen geológico. Por el contrario, en el período 1990-2009, el 80,1\% de las pérdidas de vidas humanas se debió a desastres de origen climático. Es muy probable que este comportamiento se revierta en la presente década debido a las muertes causadas por el terremoto en Haití (2010).

En el caso de Centroamérica, los eventos climáticos causaron el 49,2\% de los fallecimientos atribuidos a desastres, mientras que los geológicos produjeron el 48,9\% de las muertes. Nótese que en esa región se registraron muertes en el $65 \%$ de los desastres de origen climático, en los que fallecieron, en promedio, 216 personas. En el caso del Caribe, los eventos geológicos produjeron el 92,2\% de las

12 A lo largo de este artículo, al hablar de desastre se hace referencia a la ocurrencia de un desastre en un país. Si un evento, como el huracán Mitch, afecta a varios países, se realiza una observación por cada uno de los países afectados.

${ }^{13}$ En el mundo, diez desastres ocasionaron el 53,8\% de todas esas muertes. El único desastre ocurrido en América Latina y el Caribe incluido entre ellos es el terremoto de Puerto Príncipe de 2010.

14 Cavallo y Noy (2010) sostienen que el 96\% de las muertes ocasionadas por desastres en el período 1970-2008 ocurrieron en África, América Latina y el Caribe y Asia, cuya población constituye el 75\% de la población mundial. Stromberg (2007) sostiene que en el período 1980-2004, por cada muerte causada por desastres en los países de altos ingresos, ocurrieron 12 muertes en los países de bajos ingresos. Estos resultados derivan de cuatro eventos que causaron la mayoría de las muertes: las sequías de 1984 en el Sudán y Etiopía, el ciclón de 1991 en Bangladesh y el tsunami de 2004 en el océano Índico. 
muertes, mientras que los climáticos causaron el 5,4\% de los fallecimientos. Se registraron muertes en el $56,6 \%$ de los eventos de este tipo, en que fallecieron 63 personas en promedio. Cabe destacar el importante incremento que se registró en el número de muertes por cada 1.000 habitantes causadas por desastres geológicos en el período 2010-2011. Este resultado está asociado al terremoto en Haití en 2010, que fue el evento en que se produjeron más muertes en la región.

\section{Población afectada}

En el período comprendido entre 1970 y 2009, 6.308 millones de personas en el mundo fueron afectadas por desastres y su número aumentó en los dos tipos de desastres climáticos considerados, lo que contrastó con la población afectada por desastres geológicos, que registró alternadamente crecimiento y decrecimiento a lo largo de esas décadas ${ }^{15}$. El $89 \%$ de los afectados vivía en Asia, mientras que el 3,3\% vivía en América Latina y el Caribe. Destaca el hecho de que la gran mayoría de las personas afectadas lo fueron por desastres de origen climático, lo que contrasta con las personas fallecidas. En el mundo, el $97 \%$ de la población afectada correspondió a eventos climáticos, mientras que el 2,7\% correspondió a eventos geológicos. En América Latina y el Caribe, estos porcentajes fueron del $83,9 \%$ y el $14,9 \%$, respectivamente ${ }^{16}$.

Otra medida que muestra la intensidad de un desastre es el número de personas afectadas por cada 1.000 habitantes en los países donde se produjeron desastres. La evidencia basada en esta medida indica que los desastres geológicos y las tormentas han sido más intensos en el Caribe y en Centroamérica que en América del Sur (véase el cuadro 3).

Cuadro 3

América Latina y el Caribe: afectados por desastres, 1970-2009a

(En número de afectados por cada 1.000 habitantes)

\begin{tabular}{|c|c|c|c|c|}
\hline Década & Tormentas & $\begin{array}{l}\text { Otros desastres de } \\
\text { origen climático }\end{array}$ & $\begin{array}{l}\text { Desastres de origen } \\
\text { geológico }\end{array}$ & Total \\
\hline \multicolumn{5}{|l|}{ El Caribe ${ }^{b}$} \\
\hline $1970-1979$ & 16,1 & 16,6 & 20,8 & 24,2 \\
\hline 1980-1989 & 30,9 & 11,5 & 0,0 & 28,1 \\
\hline 1990-1999 & 17,0 & 20,8 & 94,8 & 28,2 \\
\hline $2000-2009$ & 34,1 & 2,4 & 3,4 & 33,7 \\
\hline Total & 24,5 & 12,8 & 29,8 & 28,5 \\
\hline \multicolumn{5}{|l|}{ Centroamérica $^{\mathrm{C}}$} \\
\hline 1970-1979 & 21,7 & 4,2 & 62,9 & 60,5 \\
\hline 1980-1989 & 4,6 & 7,2 & 6,3 & 9,4 \\
\hline 1990-1999 & 14,9 & 12,4 & 3,2 & 21,4 \\
\hline $2000-2009$ & 4,9 & 13,3 & 10,2 & 21,0 \\
\hline Total & 11,5 & 9,3 & 20,6 & 28,1 \\
\hline
\end{tabular}

15 En el mundo, el cociente entre el número de personas afectadas y de muertes por causa de desastres fue 1.826. Con respecto a los desastres de origen geológico y a los de origen climático, esa razón fue 3.178 y 128, respectivamente.

${ }^{16}$ Cabe destacar que en el período 1980-2009, más del 90\% de las personas afectadas lo fueron por desastres de tipo climático. 
Cuadro 3 (conclusión)

\begin{tabular}{lcccc}
\hline Década & Tormentas & $\begin{array}{c}\text { Otros desastres de } \\
\text { origen climático }\end{array}$ & $\begin{array}{c}\text { Desastres de origen } \\
\text { geológico }\end{array}$ & Total \\
\hline América del Sur $^{d}$ & & & & 20,1 \\
\hline 1970-1979 & 0,8 & 14,8 & 24,7 & 20,8 \\
$1980-1989$ & 0,5 & 23,7 & 2,5 & 7,5 \\
$1990-1999$ & 0,6 & 7,0 & 2,8 & 6,9 \\
2000-2009 & 0,3 & 6,2 & 2,2 & 13,8 \\
\hline Total & 0,5 & 12,9 & 8,1 & \\
\hline
\end{tabular}

Fuente: Base de Datos Internacional sobre Desastres (EM-DAT) de la Oficina de Asistencia de Desastres en el Extranjero y el Centro de Investigación sobre la Epidemiología de los Desastres (OFDA/CRED) de la Universidad Católica de Lovaina.

a En 2010, en el Caribe la población afectada por eventos de origen geológico fue de 3.700 .000 personas; por tormentas, 107.200 personas, y por otros eventos climáticos, 59.200 personas. En Centroamérica estas cifras ascendieron a 1.800, 436.800 y 170.900 personas, respectivamente.

b El Caribe incluye: Antigua y Barbuda, Bahamas, Barbados, Cuba, Dominica, Granada, Haití, Jamaica, República Dominicana, Saint Kitts y Nevis, Santa Lucía, San Vicente y las Granadinas y Trinidad y Tabago.

c Centroamérica incluye: Belice, Costa Rica, El Salvador, Guatemala, Honduras, Nicaragua y Panamá.

d América del Sur incluye: Argentina, Bolivia (Estado Plurinacional de), Brasil, Chile, Colombia, Ecuador, Guyana, Paraguay, Perú, Suriname, Uruguay y Venezuela (República Bolivariana de).

\section{Daños}

En el período 1970-2011, los daños causados por desastres climáticos representaron el 72,9\% del total mundial de los daños por desastre, mientras que los causados por desastres de origen geológico representaron el $27 \%{ }^{17}$. En el caso de los primeros, las tormentas ocasionaron el $53,4 \%$ de los daños, mientras que otros desastres climáticos provocaron el 46,6\% de estos. Cabe destacar que el 9,1\% del total de los daños se produjo en América Latina y el Caribe.

La base de datos EM-DAT, a pesar de ser la que lleva el mejor registro de desastres en el mundo, subestima los daños por este concepto debido a que solamente se registraron daños en un $32,1 \%$ de los eventos incluidos en ella ${ }^{18}$. Si se desglosa esta cifra por el tipo de amenaza que los origina, se observa que solamente se registraron daños en el caso del $32,3 \%$, el $50,8 \%$ y el $29,3 \%$ de los desastres geológicos, las tormentas y otros desastres climáticos, respectivamente. Dos elementos parecen explicar entonces la preponderancia de los daños ocasionados por los desastres de tipo climático: el aumento de este tipo de eventos en el mundo con respecto a los de origen geológico y el mayor porcentaje de eventos de este tipo con registro de daños. Sin embargo, cabe destacar que el daño promedio por evento geológico fue mayor que el daño promedio por evento climático. Un resultado similar obtuvieron Bello, Ortiz y Samaniego (2014) utilizando la base de datos Estimaciones de Impacto de Desastres realizadas por la CEPAL, 1972 a 2010 (de uso interno).

En lo que se refiere a los daños, más que hacer referencia a valores absolutos, vale la pena expresarlos como porcentajes del PIB de los países que fueron afectados por desastres en cada región y cada año. Nótese que el Caribe es la región donde los daños por desastres, en promedio, han representado un mayor porcentaje del PIB, superando el $8 \%$ en seis ocasiones. Le sigue Centroamérica, donde los daños causados por desastres superaron el 8\% del PIB en dos ocasiones.

\footnotetext{
17 La pregunta que surge al observar esta evolución es “¿cuánto de esto es atribuible al cambio climático?”. Una respuesta fue brindada en el informe especial del Grupo Intergubernamental de Expertos sobre el Cambio Climático (IPCC, 2012). Este panel señala que hasta ahora las tendencias de daños de desastres ajustadas por riqueza y población no son atribuibles al cambio climático.

18 Nótese que este porcentaje ha sido similar en las cuatro décadas consideradas: un 35,4\%, un 29,9\%, un 39,4\% y un $28,1 \%$, respectivamente. En lo que respecta a América Latina y el Caribe, los porcentajes fueron del 38,7\%, el 31,1\%, el 34,0\% y el $25,8 \%$, respectivamente.
} 
En América Latina y el Caribe, este indicador llegó a igualar o superar el 1\% del PIB de los países afectados solamente en dos ocasiones.

Resumiendo, las dos subregiones más afectadas por desastres, tanto en lo que respecta a la población como a la incidencia material, son el Caribe y Centroamérica. Comparadas con América del Sur y México, son territorios menos extensos y tienen menor número de habitantes. Cabe recordar que una característica de los desastres es que en la mayoría de los casos solo afectan un área, una región o un departamento específico. La excepción es el Caribe, donde algunos huracanes han afectado completamente a islas pequeñas. Tal como se describe en Albala-Bertrand (1993), los desastres están confinados a un determinado espacio y afectan indirectamente al resto de la economía a través de los vínculos del sistema local con el sistema nacional. Cuanto más fuertes sean estos vínculos, mayor será el potencial de transmisión. Cuando el efecto del desastre se compara con algún indicador económico nacional como, por ejemplo, el PIB, no se aprecia su verdadera dimensión en el ámbito de la economía regional, que en la práctica es la que puede recibir el mayor impacto, y en países grandes esto puede verse minimizado, como puede observarse en el gráfico 2A.

Gráfico 2

América Latina y el Caribe: daños causados por desastres, 1980-2010

(En porcentajes del PIB)

\section{A. América Latina y el Caribe ${ }^{a}$}



B. El Caribe ${ }^{b}$




Gráfico 2 (conclusión)



Fuente: Base de Datos Internacional sobre Desastres (EM-DAT) de la Oficina de Asistencia de Desastres en el Extranjero y el Centro de Investigación sobre la Epidemiología de los Desastres (OFDA/CRED) de la Universidad Católica de Lovaina.

Nota: PIB: Producto interno bruto.

a América Latina y el Caribe incluye: Antigua y Barbuda, Argentina, Bahamas, Barbados, Belice, Bolivia (Estado Plurinacional de), Brasil, Chile, Colombia, Costa Rica, Cuba, Dominica, Ecuador, El Salvador, Granada, Guatemala, Guyana, Haití, Honduras, Jamaica, México, Nicaragua, Panamá, Paraguay, Perú, República Dominicana, Saint Kitts y Nevis, Santa Lucía, San Vicente y las Granadinas, Suriname, Trinidad y Tabago, Uruguay y Venezuela (República Bolivariana de).

b El Caribe incluye: Antigua y Barbuda, las Bahamas, Barbados, Cuba, Dominica, Granada, Haití, Jamaica, República Dominicana, Saint Kitts y Nevis, Santa Lucía, San Vicente y las Granadinas y Trinidad y Tabago.

c Centroamérica incluye: Belice, Costa Rica, El Salvador, Guatemala, Honduras, Nicaragua y Panamá.

\section{Impactos económicos de los desastres}

Como se mencionó, los desastres son una perturbación que podría afectar diversas variables económicas como el PIB, las finanzas públicas y los precios, y variables sociales como el índice de pobreza. El impacto en la primera variable ha sido estudiado extensamente ${ }^{19}$. Un análisis pionero en el tema es el de Albala-Bertrand (1993), quien estudia los impactos de corto plazo de los desastres. Este autor utilizó datos de 28 desastres de distinto origen ocurridos en 26 países entre 1960 y 1979 y concluyó que tuvieron un impacto positivo del $0,4 \%$ en el crecimiento del PIB $^{20}$. Por su parte, Rasmussen (2004), usando una metodología similar para examinar una muestra de 12 grandes desastres que tuvieron lugar en países de la Unión Monetaria del Caribe Oriental en el período 19702002, observó que en el año en que ocurre el desastre hay un impacto negativo en el PIB. Desde el punto de vista de las cuentas fiscales se produjo un deterioro del balance fiscal debido a una caída de los ingresos y un incremento del gasto ${ }^{21}$. Las críticas realizadas a ambos trabajos son similares: las muestras son limitadas y las conclusiones no están basadas en un método estadístico formal.

En respuesta a estos cuestionamientos surgieron un conjunto de investigaciones que utilizan tanto una base de datos más amplia, la base EM-DAT, como métodos estadísticos formales.

Entre esos trabajos destaca el de Noy (2007), en el que se utilizó el método de estimación de tres etapas de Hausman y Taylor sobre una base de datos que incluye una muestra de países

\footnotetext{
19 Véase una revisión detallada de todos los aspectos económicos del tema de desastres en Cavallo y Noy (2010).

20 Se incluyen terremotos, tsunamis, ciclones, inundaciones y sequías; se consideran fundamentalmente países de África, Asia y América Latina, además de un país europeo; las estimaciones de impacto usadas por el autor indican que hubo daños, esto es, destrucción de acervo.

${ }^{21}$ Dos Reis (2004) presenta costos agregados para los países de la Unión Monetaria del Caribe Oriental en el período 1970-2000 como justificación de un mecanismo de seguro fiscal en los países de esa región.
} 
desarrollados y en desarrollo en el período comprendido entre 1970 y 2003. Como resultado de este estudio, se observó que en el corto plazo los desastres tienen un impacto adverso en la tasa de crecimiento del PIB. Después de un desastre de similar magnitud, las economías en desarrollo, sobre todo las más pequeñas, muestran caídas mayores que las de los países desarrollados. Un mejor marco institucional y mejores condiciones crediticias en los países desarrollados estarían entre las causas que permitirían explicar este resultado, así como el hecho de que en un país pequeño es más probable que los desastres tengan una dimensión nacional, como es el caso de los países del Caribe.

En otros trabajos se estudiaron los impactos de diferentes desastres en la actividad económica en el corto y en el largo plazo. Raddatz (2009) utiliza técnicas de series de tiempo en panel (modelo autorregresivo de rezagos distribuidos (PARDL) y modelo de vectores autorregresivos (PVAR)) para estimar los efectos de corto y largo plazo en el PIB de los desastres climáticos controlando por otras variables. En su muestra incluye países que han sufrido al menos un desastre climático mayor desde 1950. Este autor concluye que los desastres tienen impactos estadísticamente significativos en el producto, provocando una disminución del PIB per cápita de un 1\%, que es mayor que un impacto típico de una perturbación en los términos de intercambio (considerados importantes fuentes de fluctuación). El efecto acumulativo de un desastre climático es de 0,6 puntos porcentuales del PIB per cápita (0,5 puntos porcentuales en el primer año). Los desastres geológicos no presentaron impactos estadísticamente significativos en el PIB per cápita. Este resultado, al igual que los de otros trabajos, muestra que tratar los desastres como un agregado puede inducir a error.

Igualmente, Loayza y otros (2009), utilizando un estimador de panel dinámico (método generalizado de momentos (MGM)), para una muestra de 94 países en desarrollo y desarrollados en el período 1961-2005, observaron que: i) un indicador generalizado de desastres no afecta la tasa de crecimiento del PIB, mientras que, si se consideran diferentes tipos de desastres por separado, solo el impacto de las inundaciones fue estadísticamente significativo y positivo; ii) con respecto a la tasa de crecimiento de distintos sectores como la agricultura, la industria y los servicios, las sequías y las tormentas afectan negativamente al primero, mientras que las inundaciones lo hacen positivamente ${ }^{22}$. No hay impactos estadísticamente significativos de ningún tipo de desastres en el PIB industrial. Por su parte, la tasa de crecimiento del sector del comercio registró un resultado positivo ante las inundaciones. En lo que respecta a la muestra de los países en desarrollo, los únicos tipos de desastres que afectaron a la tasa de crecimiento del PIB fueron las sequías (negativamente) y las inundaciones (positivamente). Estos resultados se verifican tanto en el caso de la tasa de crecimiento del PIB industrial como en el caso de la tasa de crecimiento del PIB agrícola. Además, en el caso del PIB industrial también las tormentas y los terremotos afectaron su dinámica de manera positiva. Por último, ningún tipo de desastre tuvo un impacto estadísticamente significativo en el sector de los servicios.

En el mismo sentido, Jaramillo (2009), quien estimó regresiones del tipo de las realizadas por Islam (1995) para analizar desastres clasificados según su incidencia, concluye que los desastres, sobre todo los climatológicos, tienen un impacto de corto plazo, de entre dos y cinco años. Igualmente establece que solo en un reducido grupo de países (aquellos que han sido afectados significativamente por desastres) se producen impactos permanentes en la tasa de crecimiento del PIB per cápita.

Por su parte, Hausmann, Rodríguez y Wagner (2006), en su estudio sobre los colapsos de crecimiento, concluyen que los desastres no son estadísticamente significativos para explicar la probabilidad de que en un país se produzca un retroceso temporal del PIB per cápita. Otros eventos como guerras, transiciones políticas, caídas abruptas de las exportaciones o reducciones repentinas de los flujos de capital resultaron estadísticamente significativos para determinar esa variable. En este punto es relevante el estudio de Cavallo y otros (2010), basado en el uso de la metodología conocida

\footnotetext{
22 Con respecto a la afectación sectorial de los distintos tipos de desastres en términos de conceptos de metodología de daños y pérdidas, véase Bello, Ortiz y Samaniego (2014).
} 
como estudio de evento comparativo, en que se concluye que incluso en el caso de grandes desastres tampoco hay un impacto de largo plazo en el crecimiento económico. En los únicos casos en que se observaron impactos de largo plazo - Nicaragua y la República Islámica del Irán - el resultado estuvo asociado a que, pocos años después de producirse el evento, tuvieron lugar hechos políticos de importancia que condujeron a un cambio de régimen. Estos autores no establecen una relación de causalidad entre los dos desastres considerados y los cambios políticos que los sucedieron ${ }^{23}$.

Otros trabajos en ese sentido son el de Cuaresma, Hlouskova y Obersteiner (2008) y el de Raddatz (2007). Los primeros estimaron, a partir de una muestra de 49 países, un modelo de gravedad y observaron que, luego de la ocurrencia de un desastre, solamente los países con cierto nivel de desarrollo logran mejorar su acervo de capital. Por su parte, Raddatz (2007), utilizando un modelo PVAR, estimó el efecto de las perturbaciones exógenas en el PIB de 40 países clasificados como de bajos ingresos por el Banco Mundial durante el período 1965-1997. Las perturbaciones exógenas consideradas aparte de los desastres son fluctuaciones en el precio de productos básicos, en las tasas financieras internacionales y en el nivel de actividad económica de los países desarrollados. Los resultados muestran que, aun cuando estas perturbaciones externas tienen un pequeño pero significativo impacto en el PIB per cápita de los países de bajos ingresos, solo permiten explicar una pequeña parte de la varianza total del PIB per cápita de esos países. Incluso en el largo plazo no permiten explicar más de un $11 \%$ de la varianza. El restante $89 \%$ obedece a factores que no forman parte del conjunto de variables exógenas consideradas, es decir, factores internos como conflictos, inestabilidad política y mal manejo económico.

En resumen, en esta literatura no hay consenso en cuanto al signo del impacto de corto plazo que causan los desastres o alguna desagregación de ellos sobre la tasa de crecimiento del PIB ${ }^{24}$. Con respecto al largo plazo, la evidencia indica que los desastres no tienen impactos en la tasa de crecimiento del PIB. De ser este el caso, no puede considerarse a los desastres como la causa del pobre desempeño económico secular de algunos países.

Con respecto a otras variables que podrían verse afectadas por los desastres, Cavallo, Cavallo y Rigobón (2013), usando datos en tiempo real, estudiaron dos impactos típicos: las interrupciones de la oferta de bienes y los precios ${ }^{25}$. En ese estudio se consideraron dos eventos: el terremoto que tuvo lugar en Chile el 27 de febrero de 2010 y el terremoto de la costa del océano Pacífico que afectó al Japón el 11 de marzo de 2011. Los autores observaron que en Chile el número de bienes disponibles para la venta cayó un 32\% y en el Japón disminuyó un 17\%. Para realizar estas estimaciones se consideró el punto más bajo de abastecimiento, que se produjo 61 y 18 días después de los terremotos, respectivamente. Volpe y Blyde (2013), por su parte, analizan el efecto de un evento específico, el terremoto de Chile de 2010, sobre las exportaciones de ese país. Mediante el uso del estimador de diferencias en diferencias identifican un efecto negativo en esa variable, siendo el canal de transmisión la afectación de la infraestructura vial.

En relación con las finanzas públicas, Melecky y Raddatz (2011) estiman el impacto de los desastres en la sostenibilidad fiscal. Analizan cómo los gastos y los ingresos fiscales responden a diferentes tipos de desastres, y cómo estas respuestas se relacionan con la posibilidad de los gobiernos de solicitar préstamos y con la disponibilidad de fuentes de financiamiento privadas para

\footnotetext{
${ }^{23}$ Rodrik (1998) sugiere que los conflictos sociales internos son una clave para entender por qué algunos países han experimentado disminuciones en sus tasas de crecimiento después de mediados de la década de 1970. En particular, plantea que la explicación está asociada más bien a la manera en que los conflictos sociales internos interactúan con las perturbaciones externas (como las amenazas naturales), y en que las instituciones locales son capaces de manejar estos conflictos.

24 La literatura aquí referida se centra en desastres de origen geológico o climatológico. Con respecto a otros tipos de desastres, véase Olaberría (2009), quien se centra en desastres epidemiológicos.

${ }^{25}$ Los datos usados en ese trabajo provienen del proyecto de The Billion Prices Project del Instituto de Tecnología de Massachusetts. Véase [en línea] http://bpp.mit.edu.
} 
la reconstrucción privada y pública. Los resultados muestran que las tres categorías de desastres considerados hacen caer el PIB, pero estos efectos no son estadísticamente significativos. Sin embargo, observan claras consecuencias en la política fiscal luego de un desastre climático.

El impacto de un desastre en las variables económicas debería traducirse en una afectación de las variables sociales. La evidencia empírica parece indicar que los desastres tienen una incidencia negativa en la superación de la pobreza. Christiaensen y Subbarao (2005), usando datos de hogares rurales de Kenya, observan que los habitantes de zonas áridas son más vulnerables, es decir, tienen mayor probabilidad de ser pobres que los de las zonas fértiles debido a la variabilidad de las lluvias. Igualmente, Elbers, Gunning y Kinsey (2002), Lybbert y otros (2004) y Dercon (2005), mediante la utilización de datos longitudinales relativos a Zimbabwe y Etiopía, respectivamente, observan que los desastres contribuyen a que los individuos no superen la pobreza. En estos estudios se usan datos de panel de hogares, lo que puede introducir ciertos problemas econométricos asociados al error de medida o la reducción de la muestra longitudinal. Para superar estos problemas, RodríguezOreggia y otros (2013) utilizan datos a nivel municipal de México. Los autores concluyen que en los municipios donde ocurrieron desastres hay rezagos en ciertos indicadores sociales como el índice de desarrollo humano y varias medidas de pobreza. Estas conclusiones están basadas en el estimador de diferencias en diferencias.

\section{Metodología de estimación}

Siguiendo la metodología de Raddatz (2007) y de Melecky y Raddatz (2011), en este análisis se estima el impacto de los desastres en la tasa de crecimiento del PIB per cápita ajustado por la paridad del poder adquisitivo (PPA) y sobre la tasa de crecimiento del gasto del gobierno central per cápita (GG) de los países de América Latina y el Caribe a partir de datos anuales del período 1970-201026. El objetivo que se persigue es reflejar la magnitud y la duración de la respuesta de la actividad económica y de una variable de política, como el gasto fiscal, ante un desastre ${ }^{27}$. Estas estimaciones se hicieron controlando por otras variables expresadas también en tasas de crecimiento calculadas a partir de diferencias logarítmicas como el PIB de los países de altos ingresos (PIBPAl) y el índice de términos de intercambio de Deaton y Miller (DM). También se utilizó la tasa de interés internacional (R). La estimación se hace utilizando un modelo de vectores autorregresivos basado en datos de panel (PVAR).

EI PIB real per cápita ajustado por la PPA fue obtenido de Penn World Tables (versión 7.0), mientras que el gasto del gobierno per cápita fue obtenido de World Development Indicators (WDI) del Banco Mundial.

Los desastres se miden por país y por año sobre la base de la siguiente clasificación: i) geológicos (GEO), lo que incluye terremotos, deslizamientos de tierra, erupciones volcánicas y maremotos; ii) tormentas (TOR), y iii) otros desastres hidroclimáticos (OT), entre los que se cuentan inundaciones, sequías y temperaturas extremas. Para cada categoría, los desastres se miden como una variable ficticia. El criterio utilizado para definirla, es decir cuando toma el valor 1, es el siguiente: al considerar todos los desastres de una misma categoría en un año, el número total de muertes es mayor a 1 por cada 10.000 habitantes, o el porcentaje de afectados es mayor al $5 \%$ de la población, o el daño total es mayor a un $5 \%$ del PIB.

${ }^{26}$ Es importante tener en cuenta que las estimaciones realizadas en esta investigación corresponden solamente a los países de América Latina y el Caribe, una muestra más pequeña que la utilizada en trabajos referidos en la tercera sección de este artículo que se basan en datos mundiales, por lo que tendrán una mayor varianza.

27 Se prefirió la variable gasto público per cápita sobre otras variables fiscales como ingresos o deuda pública, debido a que permite reflejar mejor la reacción de los gobiernos ante perturbaciones como un desastre. 
Las otras variables utilizadas en la estimación tienen por objeto controlar por otras perturbaciones que puedan afectar a los países de América Latina y del Caribe, como el desempeño de la economía mundial, la evolución de los términos de intercambio y el comportamiento del mercado financiero internacional. Con respecto al primero, se utilizó la tasa crecimiento del logaritmo del PIB de los países de altos ingresos definidos en la base de datos World Development Indicators del Banco Mundial. En lo que se refiere a la evolución de los términos de intercambio se construyó para cada país el índice Deaton-Miller siguiendo la metodología expuesta en Deaton y Miller (1996), que captura las fluctuaciones de los precios de los productos básicos más importantes de la economía de cada país. Los precios de los productos básicos se obtuvieron de las series de datos proporcionadas por la Conferencia de las Naciones Unidas sobre Comercio y Desarrollo (UNCTAD), mientras que las estadísticas de comercio exterior de cada país se obtuvieron de la Base de Datos Estadísticos de las Naciones Unidas sobre el Comercio de Productos Básicos (COMTRADE). Por último, para aproximar las condiciones financieras internacionales se utilizó la tasa de interés de los bonos del Gobierno de los Estados Unidos.

Para un país $i$, nuestro modelo estructural corresponde a:

$$
A_{0} x_{i, t}=\alpha_{i}+\beta_{i} t+\sum_{j=1}^{q} A_{j} x_{i, t-j}+\varepsilon_{i, t}
$$

Donde

$$
\begin{gathered}
x_{i, t}=\left(z_{i, t}^{\prime}, y_{i, t}^{\prime}\right) \\
z_{i, t}^{\prime}=\left(T O R_{i, t}, O T_{i, t}, G E O_{i, t}, P I B P A I_{t}, D M_{i, t}, R_{t}\right) \\
y_{i, t}^{\prime}=\left(P I B_{i, t}, G G_{i, t}\right)
\end{gathered}
$$

Donde (3) y (4) son los vectores de variables exógenas y endógenas del modelo, respectivamente. Nótese que seis variables, TOR, OT, GEO, DM, PIB y GG, tienen los subíndices $i$ y $t$ debido a que corresponden al país $i$ y varían a través del tiempo $t$. Las otras dos variables, PIBPAl y R, tienen solo el subíndice $t$ debido a que son comunes a todos los países de la muestra y su variación se produce a través del tiempo.

El supuesto de identificación principal de este trabajo es que las variables del vector $Z$ no responden a las variables en el vector $Y$ en ningún rezago, lo que equivale a imponer una estructura diagonal en bloque a las matrices A. Lo anterior significa que la ocurrencia de desastres, el PIB de los países de altos ingresos, los términos de intercambio y la tasa de interés internacional no se ven influidos por el desempeño económico pasado de los países de América Latina y el Caribe ni por el gasto del gobierno per cápita, pero todas estas variables probablemente tengan efectos contemporáneos o rezagados en el desempeño económico.

El supuesto de que las matrices A son diagonales en bloque nos permite identificar el efecto de cada variable del vector $Z$ en las variables en el vector $Y$, pero identificar el impacto de las variables en el vector $Z$ requiere supuestos más fuertes. Primeramente se asume que la ocurrencia de los desastres es completamente exógena, es decir, que no solo no está relacionada con las variables en el vector $Y$, sino que tampoco está relacionada con las otras variables en el vector $Z$. Para el resto de las variables en el vector $Z$ se impone una estructura triangular inferior en la matriz $A$. En la especificación propuesta se asume que la ordenación va del PIB de los países de altos ingresos al índice de términos de intercambio y a la tasa de interés internacional. 
Se supone que la dinámica de este modelo, representada por las matrices A, es común a todas las unidades del corte transversal, esto es, a cada país. La razón de este supuesto es que, dado el tamaño de la dimensión temporal de los datos, no es posible estimar dinámicas específicas para cada país a menos que se reduzca considerablemente el número de variables exógenas incluidas o el número de rezagos considerados. En cuanto al número de rezagos, los contrastes utilizados para determinar su número óptimo arrojaron como resultado entre dos y tres rezagos. Debido al tamaño de la base de datos se emplearon dos en las estimaciones del modelo PVAR ${ }^{28}$.

A partir de todos estos supuestos se estiman las funciones impulso respuesta (FIR) para la tasa de crecimiento del PIB per cápita y la tasa de crecimiento del gasto del gobierno per cápita ante cambios en cada una de las variables que pertenecen al vector Z. Las FIR se calculan para un período de cinco años. Se realizaron estimaciones relativas a tres muestras: i) la muestra completa, esto es, para los países de América Latina y el Caribe; ii) para los países del Caribe, y iii) para los países de Centroamérica ${ }^{29}$.

\section{Estimaciones}

\section{Funciones impulso-respuesta de la tasa de crecimiento del PIB per cápita}

En lo que se refiere a la muestra completa y para las dos subregiones consideradas, en los gráficos 3,4 y 5 pueden apreciarse las funciones impulso respuesta (FIR) de la tasa de crecimiento del PIB per cápita ante perturbaciones en cada una de las variables del vector $z_{i, t}^{\prime}$, para un período de cinco años. Todos los gráficos se presentan siguiendo el supuesto del orden de identificación utilizado. Nótese que estos presentan las FIR ajustadas a un intervalo de confianza al 95\%.

\section{Gráfico 3}

América Latina y el Caribe: funciones impulso-respuesta del PIB (En puntos porcentuales y años)

A. Tormentas



B. Otros desastres climáticos

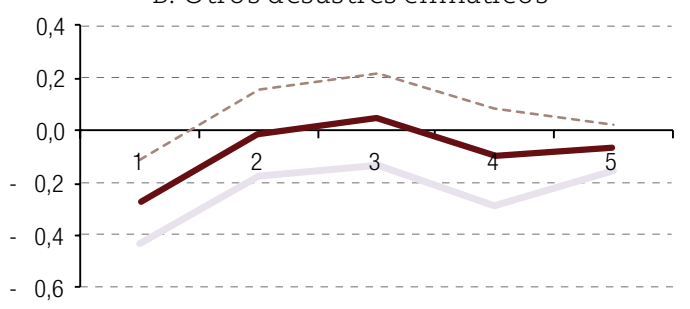

${ }^{28}$ Para revisar la robustez de los resultados fueron utilizados tres rezagos. Las FIR no cambian sustancialmente con respecto a las presentadas en la próxima sección.

29 También se hicieron estimaciones para América del Sur que por motivos de extensión no están incluidas en este documento, pero que pueden solicitarse al autor. Nótese en ese caso que la respuesta de la tasa de crecimiento del PIB per cápita fue significativa y negativa en el segundo y el tercer año ante perturbaciones en la variable otros desastres climáticos. No se consiguió ninguna respuesta estadísticamente significativa de la tasa de crecimiento del gasto del gobierno per cápita ante una perturbación en ninguna de las categorías de desastres empleadas. 
Gráfico 3 (conclusión)

C. Desastres geológicos

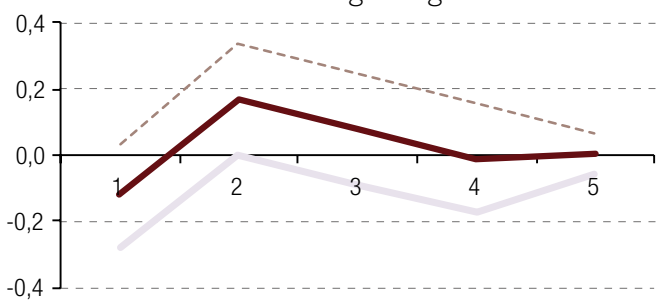

E. Términos deintercambio



D. PIB de los países de altos ingresos

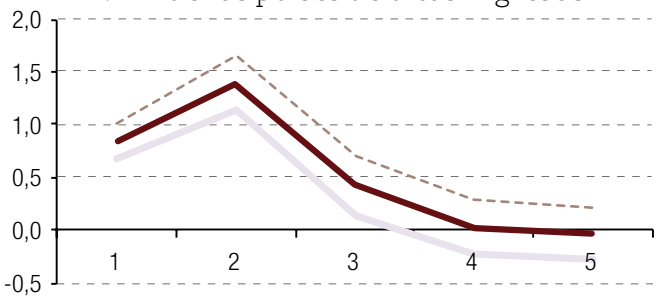

F. Tasa de interés internacional

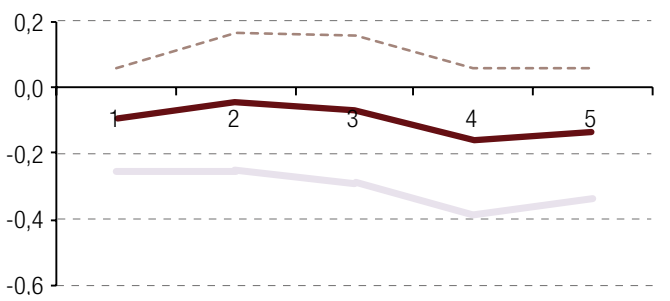

Fuente: Elaboración propia.

Nota: PIB: Producto interno bruto.

Gráfico 4

El Caribe: funciones impulso-respuesta del PIB

(En puntos porcentuales y años)

\section{A. Tormentas}

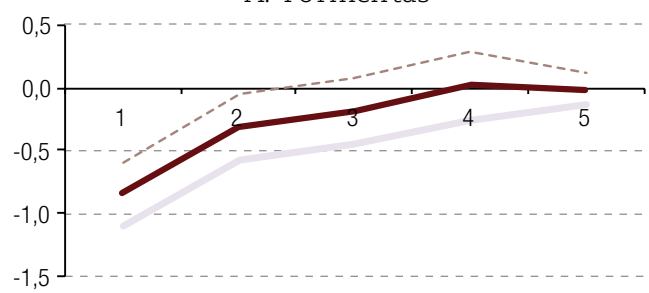

C. Desastres geológicos

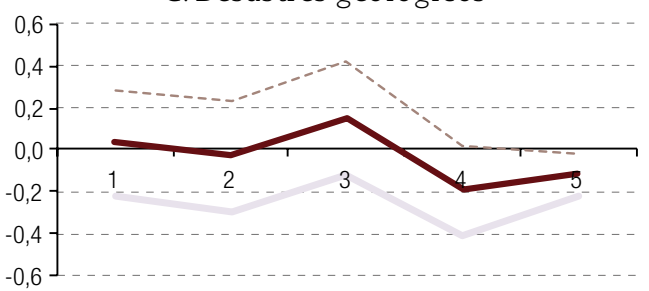

E. Términos deintercambio

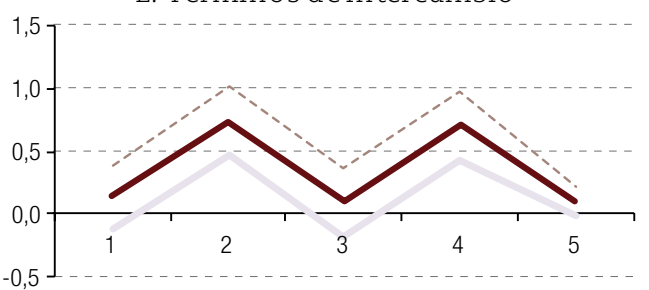

B. Otros desastres climáticos



D. PIB de los países de altos ingresos



F. Tasa de interés internacional

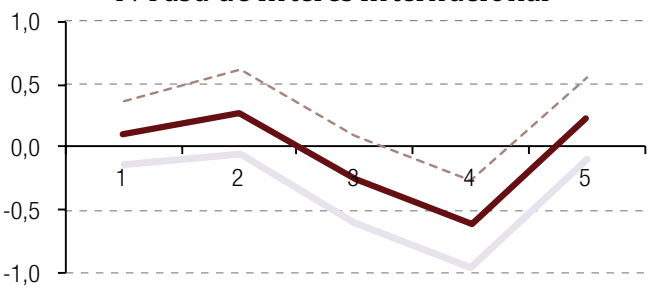

Fuente: Elaboración propia.

Nota: PIB: Producto interno bruto. 


\section{Gráfico 5}

Centroamérica: funciones impulso-respuesta del PIB

(En puntos porcentuales y años)

A. Tormentas

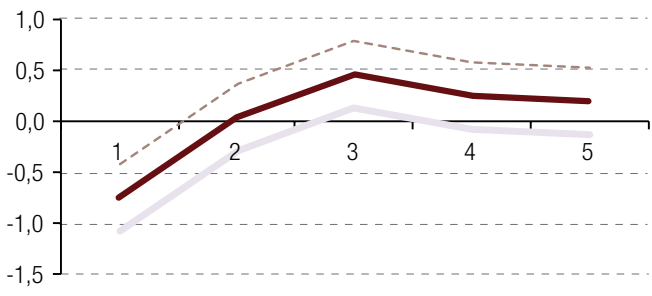

C. Desastres geológicos

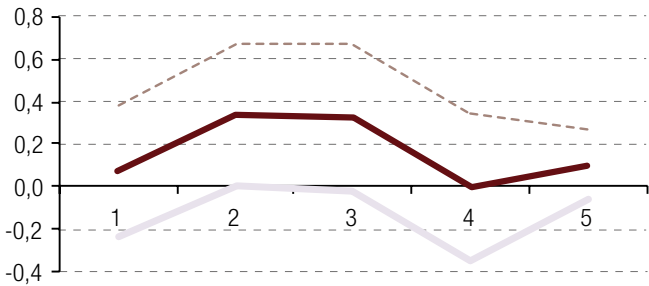

E. Términos de intercambio

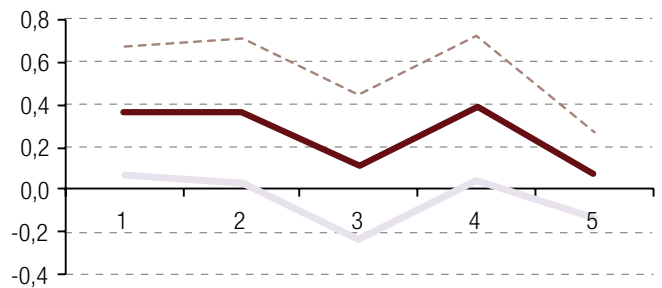

B. Otros desastres climáticos

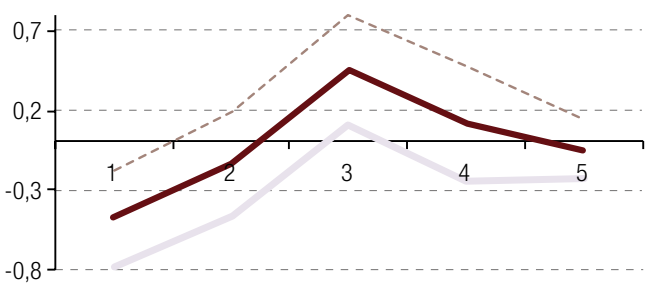

D. PIB de los países de altos ingresos

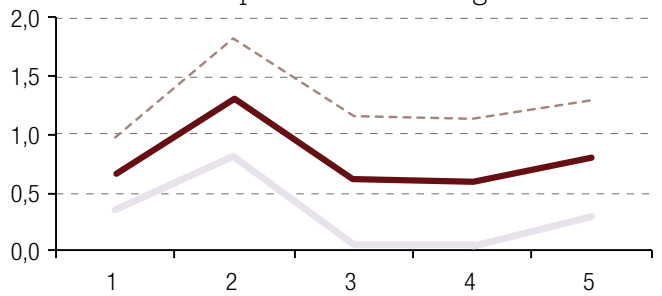

F. Tasa de interés internacional

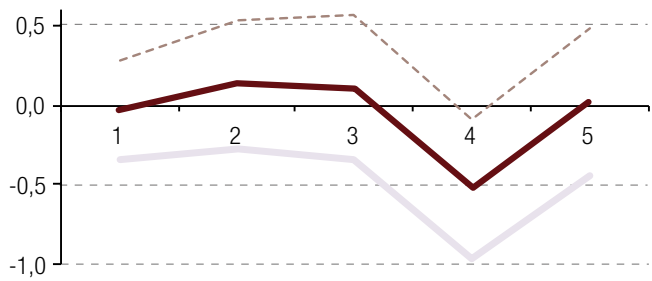

Fuente: Elaboración propia.

Nota: PIB: Producto interno bruto.

En América Latina y el Caribe, la variable tormentas tiene en el primer año un impacto negativo de aproximadamente 0,16 puntos porcentuales en la tasa de variación del PIB per cápita, que se revierte en el segundo año cuando el impacto pasa a 0,2 puntos porcentuales. Por su parte, la variable otros desastres climáticos tiene un impacto estadísticamente significativo de aproximadamente $-0,24$ puntos porcentuales en la tasa de variación del PIB per cápita y solamente para un año. En el caso de la variable desastres geológicos, este impacto es positivo, de 0,18 puntos porcentuales, en el segundo año (véanse los gráficos 3A, 3B y 3C). Las dos variables cuyas perturbaciones afectan en mayor medida la tasa de crecimiento del PIB per cápita son la tasa de variación del PIB de los países de altos ingresos y la tasa de variación del índice de términos de intercambio (véanse los gráficos 3D y 3E). Una perturbación en la tasa de variación del PIB de los países de altos ingresos tiene un efecto positivo en el crecimiento del PIB: en el primer año, este es cercano a 0,8 puntos porcentuales, llegando en el segundo año a crecer un 1,4\%, y en el tercer año aproximadamente 0,5 puntos porcentuales. A partir del cuarto año converge en cero. Por otro lado, una perturbación positiva en la tasa de variación de los términos de intercambio tiene un efecto de ese signo por cuatro años. Como era de esperar, en el primer año el crecimiento del PIB es positivo y de magnitud cercana a 0,1 puntos porcentuales. Este aumento se ve reforzado por un crecimiento aun mayor 
en el segundo año, con un valor de aproximadamente 0,7 puntos porcentuales. En el tercer año, la tasa de crecimiento disminuye, acercándose al 0,1\%, para luego subir a 0,3 puntos porcentuales. A partir del quinto año, el crecimiento del PIB per cápita converge en cero. Por último, el efecto de una perturbación en la tasa de interés internacional no es estadísticamente significativo en la tasa de crecimiento del PIB per cápita.

En las estimaciones correspondientes al Caribe se observa una evidencia más fuerte del efecto de los desastres climáticos en la tasa de crecimiento del PIB per cápita. Una perturbación en la variable tormentas causa una respuesta estadísticamente significativa y negativa en los dos primeros años, de aproximadamente 0,7 y 0,3 puntos porcentuales, respectivamente (véase el gráfico 4A). Una perturbación en la variable otros desastres climáticos tiene un efecto negativo y significativo cercano a 0,3 puntos porcentuales solamente durante un período (véase el gráfico 4B). Por su parte, las perturbaciones en los desastres geológicos no tienen un efecto significativo en el PIB de esta región. Con respecto a una perturbación en la tasa de crecimiento del PIB per cápita de los países de altos ingresos, así como en la región en su conjunto, es la de mayor efecto en la tasa de crecimiento del PIB per cápita de los países del Caribe, ya que en los dos primeros años se observan respuestas cercanas a 1 y 0,8 puntos porcentuales, respectivamente. A partir del tercer año, el efecto deja de ser estadísticamente significativo (véase el gráfico 4D). En el caso de una perturbación en la tasa de variación de los términos de intercambio, se produjeron efectos positivos los dos primeros años, de 0,1 y 0,7 puntos porcentuales, respectivamente, y el cuarto año, de 0,7 puntos porcentuales (véase el gráfico 4E). Por último, se repite el resultado para toda la región con respecto a la variación de la tasa de interés internacional.

En el caso de Centroamérica, perturbaciones tanto en la variable tormentas como en la de otros desastres climáticos generan el mismo patrón de respuesta que la tasa de variación del PIB per cápita, un decrecimiento en el primer año de aproximadamente 0,8 y 0,5 puntos porcentuales, respectivamente, y, en ambos casos, una recuperación cercana a 0,45 puntos porcentuales durante el tercer año (véanse los gráficos $5 \mathrm{~A}$ y $5 \mathrm{~B}$ ). Con respecto a los desastres geológicos no se registró un efecto significativo en el primer año, pero durante el segundo y el tercer año se alcanzó un repunte cercano a 0,4 puntos porcentuales (véase el gráfico 5C). Nótese que en el caso de los tres tipos de desastres, la convergencia se produce en el cuarto año. En las FIR con las otras variables se alcanzan resultados similares a los comentados anteriormente, esto es, la variable más importante en términos de magnitud del efecto en la tasa de crecimiento del PIB de los países centroamericanos es una perturbación en la tasa de crecimiento de las economías de altos ingresos. Nótese que ese efecto no se extingue en cinco años ${ }^{30}$.

\section{Funciones impulso-respuesta de la tasa de crecimiento del gasto del gobierno per cápita}

En este caso, el foco del análisis se centra en la respuesta de la tasa de crecimiento del gasto del gobierno per cápita a las perturbaciones en las variables de desastres y se presentan solamente las FIR correspondientes a esos eventos, que pueden verse en los gráficos 6, 7 y 8. En el caso de América Latina y el Caribe, las perturbaciones en la variable tormentas no tuvieron una respuesta estadísticamente significativa en la variable gasto del gobierno per cápita, mientras que las perturbaciones en la variable otros desastres climáticos tuvieron una respuesta positiva y estadísticamente significativa en los tres primeros años, de 0,5, 0,6 y 0,5 puntos porcentuales, respectivamente.

30 El efecto de esta perturbación converge en cero en ocho años. 


\section{Gráfico 6}

América Latina y el Caribe: funciones impulso-respuesta del gasto del gobierno per cápita (En puntos porcentuales y años)

\section{A. Tormentas}

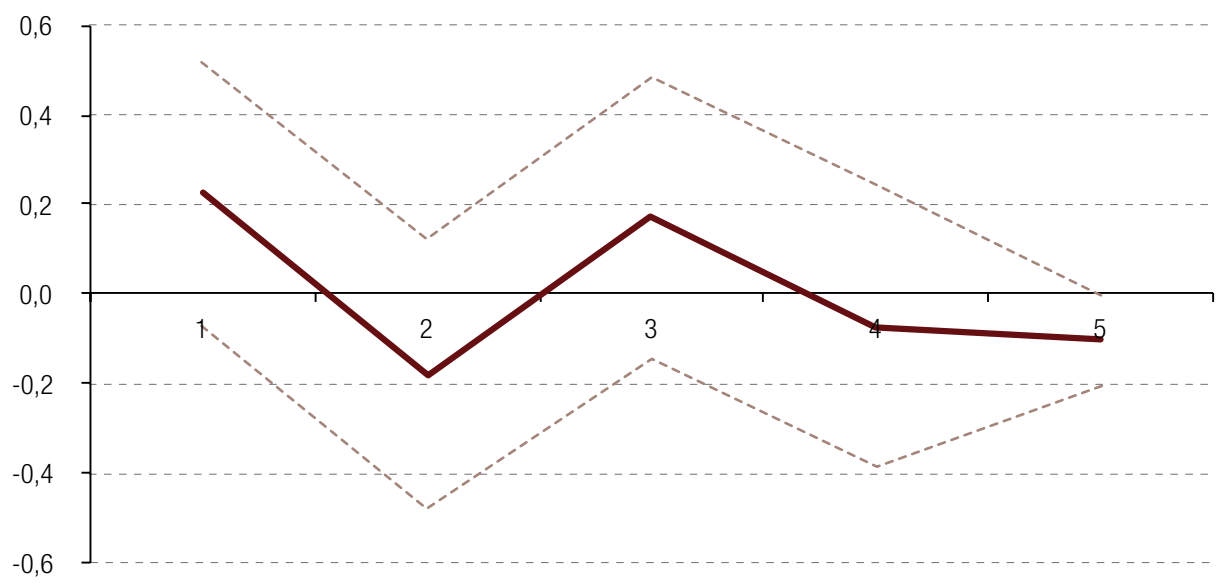

B. Otros desastres climáticos



C. Desastres geológicos

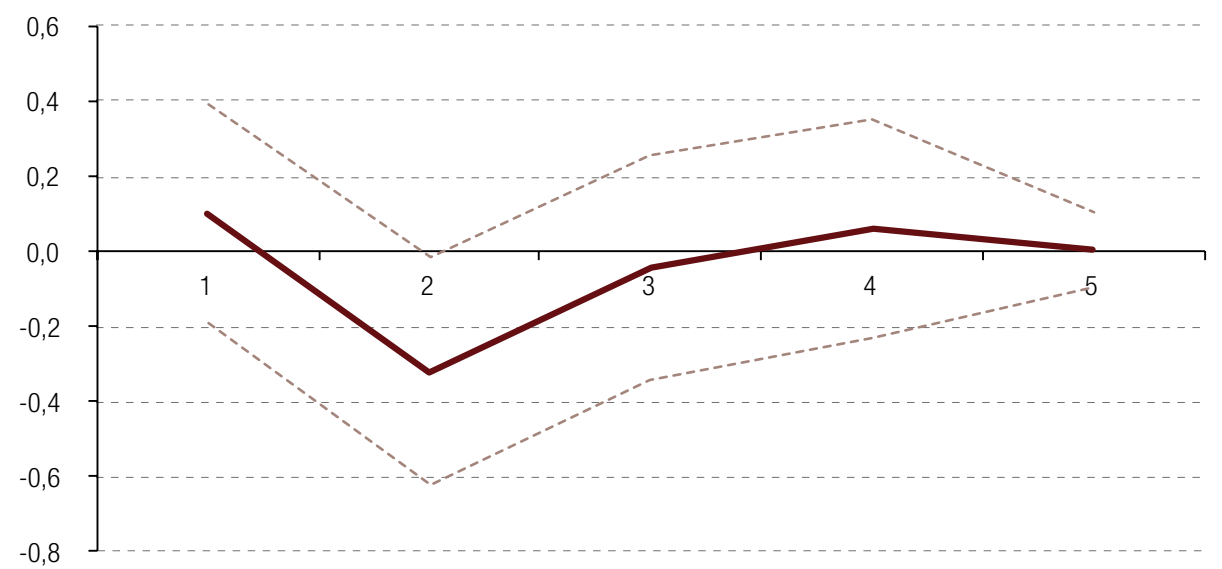

Fuente: Elaboración propia. 
En el caso de los países del Caribe, la respuesta de la tasa de crecimiento del gasto del gobierno per cápita resultó ser estadísticamente significativa ante la ocurrencia de tormentas y otros desastres climáticos en el primer año. En el caso de la variable tormentas, indujo a un aumento de dicha tasa de crecimiento de 0,7 puntos porcentuales, mientras que en el caso de la variable otros desastres climáticos esa tasa aumentó 1 punto porcentual en el primer año y 0,8 puntos porcentuales en el tercer año $^{31}$. Sin embargo, si se recuerda el efecto negativo que tanto la variable tormentas como la variable otros desastres climáticos tuvieron sobre el PIB (véase el gráfico 4), puede decirse que el incremento del gasto del gobierno no compensó la caída que el desastre causó en otros componentes de la demanda agregada. Con respecto a los desastres de origen geológico, estos no tuvieron una incidencia estadísticamente significativa en la variable exógena analizada. Este resultado puede obedecer a que, tal como se mencionó en la segunda sección de este artículo, hay muy pocos desastres de este origen en dicha región.

\section{Gráfico 7}

El Caribe: funciones impulso-respuesta del gasto del gobierno per cápita (En puntos porcentuales y años)

\section{A. Tormentas}

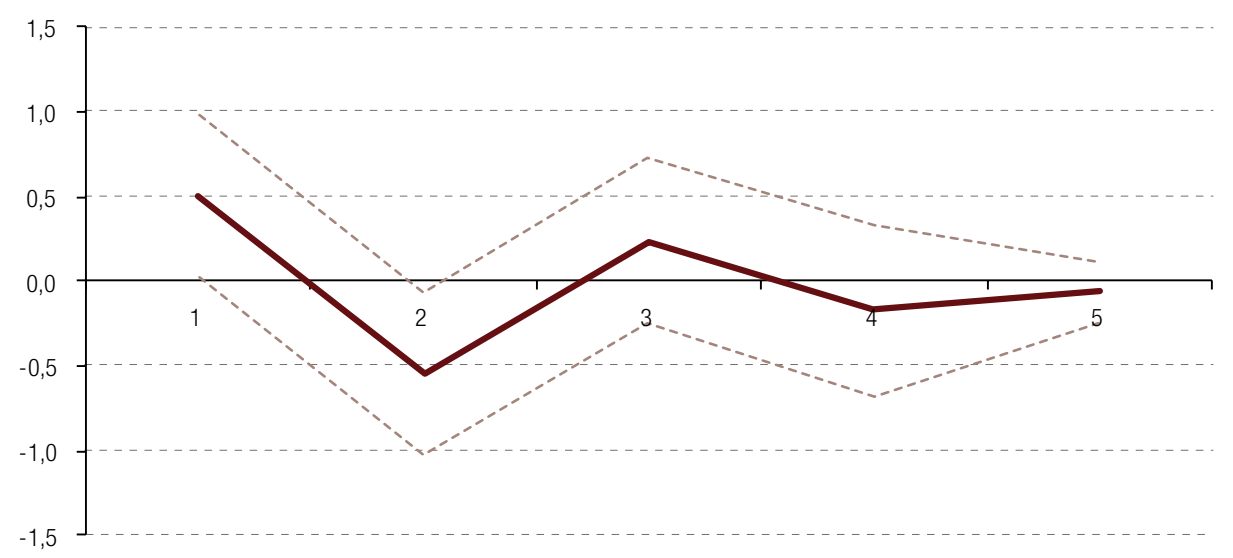

B. Otros desastres climáticos

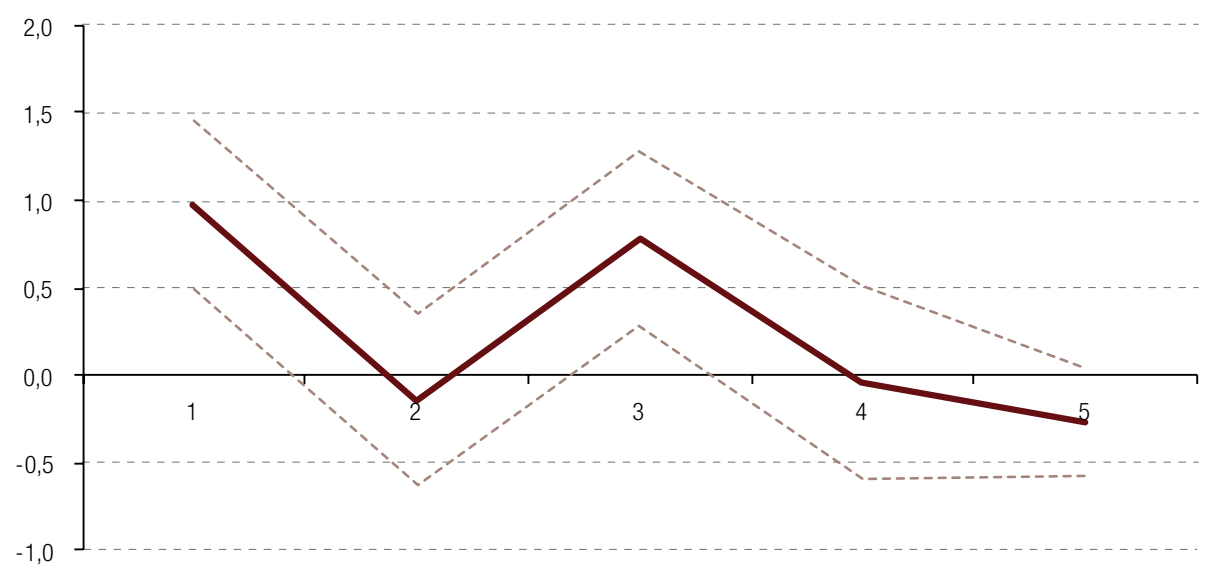

\footnotetext{
${ }^{31}$ Nótese que este resultado difiere del alcanzado respecto de toda la muestra (América Latina y el Caribe), ya que en ese caso la respuesta no resultó estadísticamente significativa.
} 
Gráfico 7 (conclusión)
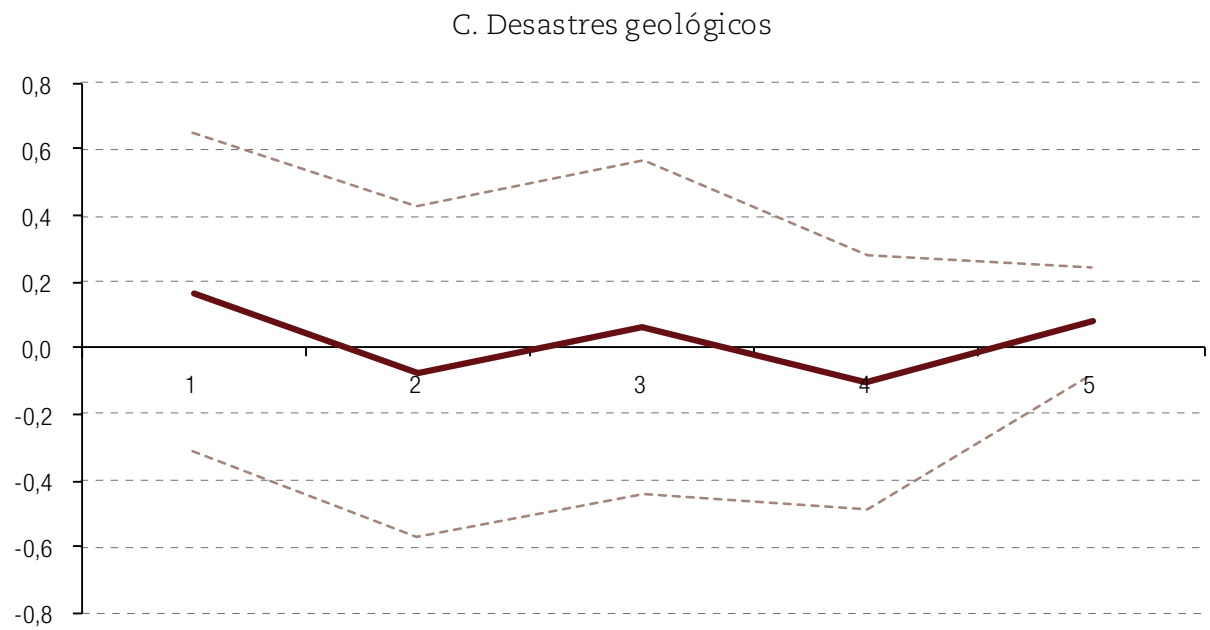

Fuente: Elaboración propia.

En el caso de Centroamérica, las FIR de la tasa de crecimiento del gasto del gobierno per cápita ante una perturbación en la variable tormentas indican que el impacto estadísticamente significativo dura dos años. Como consecuencia de esta perturbación, la tasa de crecimiento del gasto del gobierno per cápita aumentó 0,6 puntos porcentuales en el primer año y 0,8 puntos porcentuales en el segundo año. Al igual que en el caso de los países del Caribe, este resultado difiere del correspondiente a toda la muestra. En el caso de la variable otros desastres climáticos el impacto estadísticamente significativo dura tres años, registrándose aumentos de aproximadamente 0,6, 0,9 y 0,8 puntos porcentuales durante el período. Nótese que, al igual que en el caso de los países del Caribe, ese incremento del gasto no permitió compensar la disminución de otros flujos, siendo su resultado final una caída de la tasa de crecimiento del PIB. En el caso de los desastres geológicos se observó un efecto estadísticamente significativo en los dos primeros años, de aproximadamente 0,5 y 0,6 puntos porcentuales, respectivamente.

\section{Gráfico 8}

Centroamérica: funciones impulso-respuesta del gasto del gobierno per cápita

(En puntos porcentuales y años)

\section{A. Tormentas}

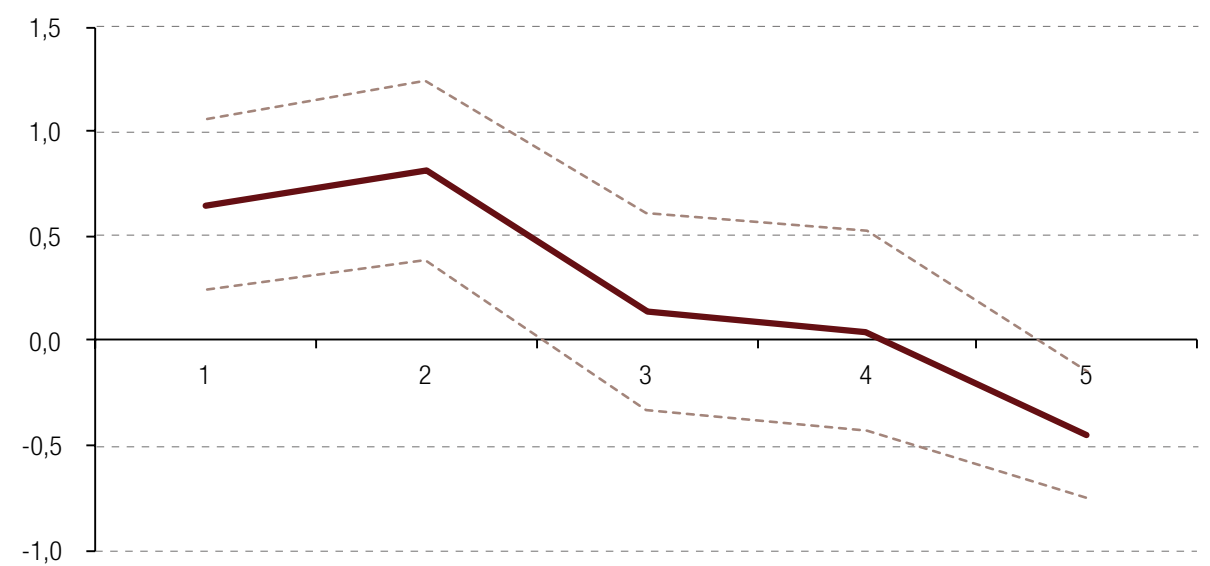




\section{B. Otros desastres climáticos}

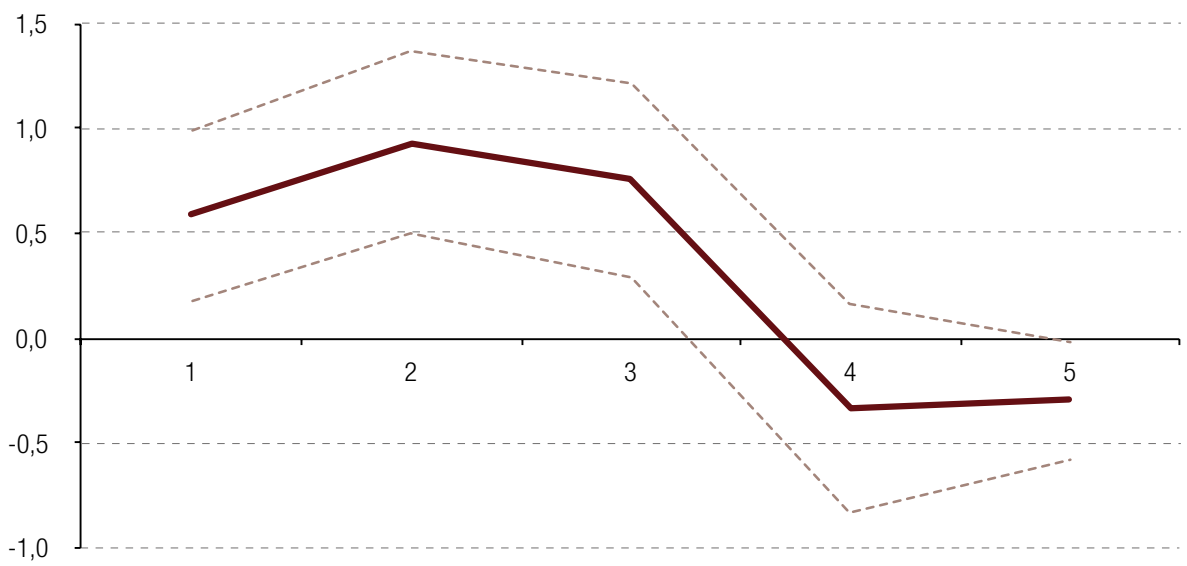

C. Desastres geológicos



Fuente: Elaboración propia.

\section{Evaluación de los resultados}

Este artículo es una contribución a la discusión sobre los impactos de los desastres en América Latina y el Caribe, sobre todo en las dos subregiones más intensamente afectadas: Centroamérica y el Caribe. Las estimaciones econométricas están focalizadas en las respuestas de la actividad económica y en una variable de política, el gasto fiscal.

Cabe destacar tres aspectos de los resultados obtenidos. En primer lugar, la respuesta tanto de la tasa de crecimiento del PIB per cápita como de la tasa de crecimiento del gasto público per cápita es mayor ante las perturbaciones en la tasa del PIB per cápita de los países de altos ingresos y ante perturbaciones en los términos de intercambio que ante las perturbaciones en las variables que aproximaron los distintos tipos de desastres. La explicación de este resultado es que las dos primeras constituyen perturbaciones que afectan a toda la economía, mientras que las otras, en la mayoría de los casos, tienen carácter local, afectan a una provincia o región del país (las excepciones han sido desastres específicos, fundamentalmente huracanes que han afectado a países enteros en el Caribe), por lo que sus impactos se ven diluidos cuando se consideran magnitudes nacionales, tanto 
por un tema de escala como por la redistribución espacial del gasto ${ }^{32}$. Así, la ayuda para atender la emergencia ocasionada por un desastre puede ser, en buena medida, adquirida en otras provincias o departamentos, por lo que la actividad sectorial, aunque sufra desajustes a escala regional, puede no experimentarlos en el nivel nacional. Si no se refleja el carácter local del desastre, la medición solo del impacto agregado estaría enmascarando el efecto en la economía regional (véase CEPAL, 2014). En muchos casos, el realce de la dimensión local está limitado por la disponibilidad de datos; por ello, la correcta medición del impacto del desastre para visibilizar la afectación de las economías locales de un país debe conllevar el uso de cuentas subnacionales, que han sido desarrolladas en algunos países de la región ${ }^{33}$. Es oportuno reiterar que, a pesar de que un desastre no tenga un impacto macroeconómico importante, sí puede tenerlo en el nivel local, afectando de manera aguda las vidas de los habitantes de los territorios donde ocurrió el evento. De ello se deriva la necesidad de elaborar políticas de gestión de riesgos de desastres en las que estén incluidos los gobiernos locales y las comunidades.

En segundo término, con respecto a los resultados derivados propiamente de los desastres, cabe destacar que la mayor duración de su impacto es de tres años. En otras palabras, pareciera que un desastre no afecta en el largo plazo el crecimiento del PIB per cápita ni el gasto fiscal per cápita de las economías de América Latina y el Caribe. Es importante destacar que se está hablando de un promedio y que el resultado también está relacionado con el modelo PVAR utilizado, en el que se supone que la dinámica es la misma para todos los países. Por último, dado el tamaño de la muestra, no se pudo verificar la robustez de este resultado considerando solamente los desastres más grandes ${ }^{34}$. Por ejemplo, Loayza y otros (2009) observaron efectos no lineales relacionados a la intensidad de los desastres.

En relación con los resultados más específicos, en el caso de los países de América Latina y el Caribe, las FIR de la tasa de crecimiento del PIB per cápita con respecto a cada uno de los tipos de desastres considerados indican que hay efectos diferenciados entre ellos, siendo negativos en el caso de perturbaciones en las variables tormentas y otros desastres climáticos, y positivos en el caso de la variable que aproxima los desastres de origen geológico. Cabe destacar que la respuesta ante la primera fue estadísticamente significativa por tres años, mientras que en el caso de las otras dos tuvo una duración de un año. Nótese que un supuesto del modelo PVAR utilizado es que se está atribuyendo la misma dinámica a cada país, lo que puede ser cuestionable dada la heterogeneidad productiva de América Latina y el Caribe. Es por ello que se procedió a hacer estimaciones para grupos de países con mayores similitudes, como los de Centroamérica y los del Caribe, que además son las dos regiones más intensamente afectadas por desastres.

Cuando se analiza el Caribe y Centroamérica, las conclusiones anteriores cambian. En los países del Caribe, la respuesta de la tasa de crecimiento del PIB per cápita a perturbaciones tanto en la variable tormentas como en la variable otros desastres climáticos fue negativa, y tuvo una duración de dos años en el primer caso y de un año en el segundo. La respuesta a un desastre de origen geológico no fue estadísticamente significativa. En los países de Centroamérica, la respuesta de la tasa de crecimiento del PIB per cápita a las tormentas y otros desastres climáticos fue similar, negativa el primer año y positiva el tercer año, y mientras que en el primer caso no fue compensada, en el segundo sí lo fue. Por su parte, la respuesta a una perturbación en la variable desastres de origen geológico fue positiva en el segundo y el tercer año. De estos resultados destaca que en ambas subregiones los dos tipos de desastres climáticos, que son los que han crecido más en las

\footnotetext{
32 Véase a este respecto la interesante discusión planteada en Albala-Bertrand (2013).

${ }_{33}$ Cabe destacar que, hasta la fecha, siete países de América Latina tienen este tipo de cuentas subnacionales: Bolivia (Estado Plurinacional de), Brasil, Chile, Colombia, Ecuador, México y Perú.

${ }^{34}$ En la base de datos utilizada en este trabajo solamente se incluyeron países de América Latina y el Caribe, por lo que es sustancialmente más reducida que la empleada en otros trabajos, como los de Loayza y otros (2009), Jaramillo (2009) y Raddatz (2007 y 2009), entre otros, que utilizaron datos de alcance mundial.
} 
últimas décadas, tuvieron en todos los casos una respuesta de la tasa de crecimiento del PIB per cápita estadísticamente significativa y negativa durante el primer año, alcanzándose una respuesta acumulada negativa en tres de cuatro casos. Esto puede ser evidencia de que, ante esos desastres, los procesos de reposición o reconstrucción de los daños pueden haber sido insuficientes. Cuaresma, Hlouskova y Obersteiner (2008) observaron que, luego de la ocurrencia de un desastre, solamente los países con cierto nivel de desarrollo logran reconstruir y lograr mejoras respecto de lo que tenían previo al evento.

Con respecto a la tasa de crecimiento del gasto público real per cápita, la FIR correspondiente a los países de América Latina y el Caribe fue estadísticamente significativa solamente en el caso de perturbación de la variable otros desastres climáticos en el primer y el tercer año. En lo que se refiere a los países del Caribe esa respuesta resultó estadísticamente significativa y positiva solamente en el caso de los desastres climáticos, en el primer año en el caso de la variable tormentas y en el primer y el tercer año en el caso de otros desastres climáticos. En cuanto a los países de Centroamérica, la respuesta ante las perturbaciones en las variables de todos los tipos de desastre fue positiva y estadísticamente significativa. En el caso de la variable tormentas esa respuesta abarcó los dos primeros años, mientras que en el caso de otros desastres climáticos se extendió por tres años. Por su parte, en el caso de los desastres geológicos esa respuesta se extendió un año. Como síntesis de estos resultados cabe destacar el incremento de la tasa de crecimiento del gasto del gobierno per cápita, que aumentó en seis de nueve casos como consecuencia de un desastre. Sin embargo, si se consideran los resultados obtenidos con relación al PIB per cápita, parecería que esta reacción no fue suficiente para evitar la caída de la tasa de crecimiento de ese indicador. Posiblemente esta reacción haya estado condicionada por limitaciones institucionales en cada país o por el grado de holgura fiscal existente antes del evento. Lo dicho constituye un fuerte argumento a favor de que los países avancen en la consolidación institucional de la política de reducción del riesgo de desastres, de manera que sea incorporada a las políticas de inversión pública.

\section{Bibliografía}

Albala-Bertrand, J. (2013), Disasters and the Networked Economy, Nueva York, Routledge. (1993), The Political Economy of Large Natural Disasters, Oxford, Clarendon Press.

Bello, O., L. Ortiz y J.L. Samaniego (2014), "La estimación de los efectos de los desastres en América Latina, 1972-2010", serie Medio Ambiente y Desarrollo, № 157 (LC/L.3899), Santiago, Comisión Económica para América Latina y el Caribe (CEPAL).

Cavallo, A., E. Cavallo y R. Rigobón (2013), "Prices and supply disruptions during natural disasters", NBER Working Paper, No 19474, Cambridge, Massachusetts, National Bureau of Economic Research.

Cavallo, E. e I. Noy (2010), "The economics of natural disasters: a survey”, IDB Working Paper Series, № 124, Washington, D.C., Banco Interamericano de Desarrollo.

Cavallo, E. y otros (2010), "Catastrophic natural disasters and economic growth", IDB Working Paper Series, № 183, Washington, D.C., Banco Interamericano de Desarrollo.

CEPAL (Comisión Económica para América Latina y el Caribe) (2014), Manual para la evaluación de desastres (LC/L.3691), Santiago.

Christiaensen, L. y K. Subbarao (2005), "Towards an understanding of household vulnerability in rural Kenya", Journal of African Economies, vol. 14, № 4, Centro de Estudios de Economía Africana.

Cuaresma, J., J. Hlouskova y M. Obersteiner (2008), "Natural disasters as creative destruction? Evidence fron developing countries", Economic Inquiry, vol. 46, № 2.

De Gregorio, J. y R. Valdés (2001), "Crisis transmission: evidence from the debt, tequila and Asian flu crises", World Bank Economic Review, vol. 25, № 2, Oxford University Press.

Deaton, A. y R. Miller (1996), "International commodity prices, macroeconomic performance and politics in Sub-Saharan Africa", Journal of African Economies, vol. 5, № 3, Centro de Estudios de Economía Africana.

Dercon, S. (2005), "Growth and shocks: evidence from rural Ethiopia", Journal of Development Economics, vol. 74, № 2, Amsterdam, Elsevier. 
Dos Reis, L. (2004), "A fiscal insurance scheme for the Eastern Caribbean Currency Union" [en línea] http:// www.iadb.org/WMSFiles/products/research/files/pubS-242.pdf.

Elbers, C., J. Gunning y B. Kinsey (2002), "Convergence, shocks and poverty", Discussion Paper, № 2002035/2, Amsterdam, Tinbergen Institute.

Hausmann, R., F. Rodríguez y R. Wagner (2006), "Growth collapses", CID Working Paper, № 136, Cambridge, Massachusetts, Universidad de Harvard.

IPCC (Grupo Intergubernamental de Expertos sobre el Cambio Climático) (2012), Managing the Risks of Extreme Events and Disasters to Advance Climate Change Adaptation, Cambridge University Press.

Islam, N. (1995), "Growth empirics: a panel data approach", Quarterly Journal of Economics, vol. 110, № 4, Oxford University Press.

Jaramillo, C. (2009), "Do natural disasters have long-term effects on growth?", Documentos CEDE, № 24, Bogotá, Universidad de los Andes.

Laeven, L. y F. Valencia (2008), "Systemic banking crises: a new database", IMF Working Paper, № 224, Washington, D.C., Fondo Monetario Internacional.

Loayza, N. y otros (2009), "Natural disasters and growth - going beyond the averages", Policy Research Working Paper Series, № 4980, Washington, D.C., Banco Mundial.

Lybbert, T. y otros (2004), "Stochastic wealth dynamics and risk management among a poor population", Economic Journal, vol. 114, № 498.

Melecky, M. y C. Raddatz (2011), "How do governments respond after catastrophes? Natural-disaster shocks and the fiscal stance", Policy Research Working Paper Series, № 5564, Washington, D.C., Banco Mundial.

Noy, I. (2007), "The macroeconomic consequences of disasters", Documento de Trabajo, № 7, Honolulu, Universidad de Hawaii.

Olaberría, E. (2009), "Economic impacts of epidemics", inédito.

Raddatz, C. (2009), "The wrath of God: macroeconomic costs of natural disasters", Policy Research Working Paper Series, № 5039, Washington, D.C., Banco Mundial.

(2007), "Are external shocks responsible for the instability of output in low-income countries?", Journal of Development Economics, vol. 84, № 1, Amsterdam, Elsevier.

Rasmussen, T. (2004), "Macroeconomic implications of natural disasters in the Caribbean", IMF Working Paper, $\mathrm{N}^{\circ}$ 04/24, Washington, D.C., Fondo Monetario Internacional.

Reinhart, C. y K. Rogoff (2008), "This time is different: a panoramic view of eight centuries of financial crises", NBER Working Paper, № 13882, Cambridge, Massachusetts, National Bureau of Economic Research.

Rodríguez-Oreggia, E. y otros (2013), "Natural disasters, human development and poverty at the municipal level in Mexico", Journal of Development Studies, vol. 49, №3, Taylor \& Francis.

Rodrik, D. (1998), "Where did all the growth go? External shocks, social conflict, and growth collapses", NBER Working Papers, № 6350, Cambridge, Massachusetts, National Bureau of Economic Research.

Skidmore, M. y H. Toya (2002), "Do natural disasters promote long-run growth?", Economic Inquiry, vol. 40, $N^{\circ} 4$, Wiley.

Stromberg, D. (2007), "Natural disasters, economic development, and humanitarian aid", Journal of Economic Perspectives, vol. 21, № 3, Nashville, Tennessee, American Economic Association.

Titelman, D., E. Pérez y R. Pineda (2009), “¿Cómo algo tan pequeño terminó siendo algo tan grande? Crisis financiera, mecanismos de contagio y efectos en América Latina”, Revista CEPAL, № 98 (LC/G.2404-P), Santiago, Comisión Económica para América Latina y el Caribe (CEPAL).

Volpe, C. y J.S. Blyde (2013), "Shaky roads and trembling exports: assessing the trade effects of domestic infrastructure using a natural experiment", Journal of International Economics, vol. 90, № 1, Amsterdam, Elsevier. 